\title{
PROJECTIVE STRUCTURE AND INTEGRABLE GEODESIC FLOWS ON THE EXTENSION OF BOTT-VIRASORO GROUP
}

\section{PARTHA GUHA}

Received 22 June 2004

To the memory of Professor Jürgen Moser

\begin{abstract}
This is a sequel to our paper (Lett. Math. Phys. (2000)), triggered from a question posed by Marcel, Ovsienko, and Roger in their paper (1997). In this paper, we show that the multicomponent (or vector) Ito equation, modified dispersive water wave equation, and modified dispersionless long wave equation are the geodesic flows with respect to an $L^{2}$ metric on the semidirect product space $\operatorname{Diff}^{s}\left(S^{1) \ltimes C} \infty\left(S^{1}\right)^{k}\right.$, where $\operatorname{Diff}^{s}\left(S^{1}\right)$ is the group of orientation preserving Sobolev $H^{s}$ diffeomorphisms of the circle. We also study the projective structure associated with the matrix Sturm-Liouville operators on the circle.
\end{abstract}

2000 Mathematics Subject Classification: 53A07, 53B50.

1. Introduction. It is known that the periodic Korteweg-de Vries (KdV) and the Camassa-Holm equation [5] can be interpreted as geodesic flow of the right invariant metric on the Bott-Virasoro group, which at the identity is given by the $L^{2}$ and the Sobolev metric $H^{1}$-inner product, respectively, [25, 26, 28, 29].

With the advancement of the integrable systems, physicists and mathematicians discovered many new multicomponent versions of the existing integrable nonlinear PDEs $[1,2,9,22]$. In the theory of integrable systems, these multicomponent generalizations have been sporadically used. There are several two-component generalizations of KdV equations, namely, Hirota-Satsuma equation [16], Wilson equation [30], and Ito equation [18] are notables among them. Almost all the integrable systems of these classes share a common property. These are all bi-Hamiltonian systems which enjoy a compatible pair of Hamiltonian structure. These systems belong to an infinite-dimensional hierarchy of bi-Hamiltonian systems. The resulting Hamiltonian flows can be mapped into each other by the recursion operator, which is formally defined as the "quotient" of the two Hamiltonian structures. Several of the well-known bi-Hamiltonian systems fall into two-component case are actually tri-Hamiltonian. There are a few disparate examples of tri-Hamiltonian systems. The classical dispersive water equation and the long waves equation [22] belong to this class of integrable systems.

In our earlier papers $[10,11]$, we have shown that the Ito equation, modified dispersive water wave equation, and modified dispersionless long waves equation arise in a unified geometric setting, all of them are integrable systems which describe geodesic flows. Thus, we unify the Ito equation, the dispersive water wave equation, and the long wave equation through a common construction, all are integrable systems which describe geodesic flows with respect to $L^{2}$ on the extension of the Bott-Virasoro group. 
Recently, there has been a growing interest in the multicomponent integrable systems. These systems are far less known than ordinary integrable systems. It was presented in [1,2] that an $N$-component system has a remarkable property of possessing $(N+1)$ local Hamiltonian structure. Antonowicz and Fordy showed that these systems were isospectral to an energy-dependent Schrödinger operator and gave a systematic derivation of the Hamiltonian from knowledge of the generalized Lax representation.

Using the extension of the Bott-Virasoro group with an $L^{2}$ metric, we will show how the multicomponent KdV-type equation arise from a geodesic flow.

Following Ebin and Marsden [8], we enlarge Diff $\left(S^{1}\right)$ to a Hilbert manifold $\operatorname{Diff}^{s}\left(S^{1}\right)$, the diffeomorphism of Sobolev class $H^{s}$. This is a topological space. If $s>n / 2$, it makes sense to talk about an $H^{s}$ map from one manifold to another. Using local charts, one can check whether the derivation of order $\leq s$ is square integrable.

The Lie algebra of $\operatorname{Diff}^{\mathcal{S}}\left(S^{1}\right) \ltimes C^{\infty}\left(S^{1}\right)$ has a three-dimensional extension (explained in the next section), namely,

$$
\operatorname{Vect}^{s}\left(S^{1}\right) \ltimes C^{\infty}\left(S^{1}\right) \oplus \mathbb{R}^{3} .
$$

Then, a typical element of this algebra would be

$$
\left(f(x) \frac{d}{d x}, u(x), \alpha\right), \quad f(x) \frac{d}{d x} \in \operatorname{Vect}\left(S^{1}\right), u(x) \in C^{\infty}\left(S^{1}\right), \alpha \in \mathbb{R}^{3} .
$$

The Diff ${ }^{s}\left(\widehat{\left.S^{1}\right) \ltimes C^{\infty}}\left(S^{1}\right)\right.$ is the nontrivial extension of $\operatorname{Diff}^{s}\left(S^{1}\right) \ltimes C^{\infty}\left(S^{1}\right)$.

In this paper, we will extend our previous results [10]. We will study the geodesic flows on $\operatorname{Diff}^{s}\left(S^{1}\right) \ltimes C^{\infty}\left(S^{1}\right)^{k}$. The Lie algebra of $\operatorname{Diff}^{s}\left(\widehat{\left.S^{1}\right) \ltimes C^{\infty}}\left(S^{1}\right)^{k}\right.$ also has a threedimensional extension

$$
\operatorname{Vect}\left(S^{1}\right) \ltimes C^{\infty}\left(S^{1}\right)^{k} \oplus \mathbb{R}^{k+2} \text {. }
$$

Motivation OF THE PAPER. This paper is focused on a wide class of $N$-component systems of nonlinear evolution equations with a hierarchy of compatible Hamiltonian structures. The compatible Hamiltonian structures are related by an integrodifferential recursion operator, but the first $N+1$ Hamiltonian structures in the hierarchy are purely differential. Examples included in this class are $N$-coupled $\mathrm{KdV}$ equations and, for $N=$ 2 , dispersive water waves, Ito's equation, and the reduced shallow water wave equations of Benney.

Our motivation is to reach how one can understand the multidimensional integrable systems as some geodesic flows or Euler-Poincaré flows on some extended Bott-Virasoro groups. This unifies several multicomponent integrable systems through a common geometrical construction and symmetry.

Organization OF THE PAPER. In Section 2, we will study the Lie algebra $\operatorname{Vect}\left(S^{1}\right) \ltimes$ $C^{\infty}\left(S^{1}\right)^{k}$ of the extended Bott-Virasoro group $\operatorname{Diff}^{s}\left(S^{1}\right) \ltimes C^{\infty}\left(S^{1}\right)^{k}$. In Section 3, we describe the projective connections on the circle, in particular, we introduce the matrix 
projective connection related to $\operatorname{Vect}\left(S^{1}\right) \ltimes C^{\infty}\left(S^{1}\right)^{k}$. In Section 4 , we study the geodesic flows on the $\operatorname{Diff}^{s}\left(S^{1}\right) \ltimes C^{\infty}\left(S^{1}\right)^{k}$ related to $L^{2}$ inner product, and this gives rise to several multicomponent integrable systems corresponding to the different hyperplanes in the dual space

$$
C^{\infty}\left(S^{1}\right) \oplus \underbrace{C^{\infty}\left(S^{1}\right) \oplus \cdots C^{\infty}\left(S^{1}\right)}_{k} \oplus \mathbb{R}^{3} .
$$

In Section 5, we study the geodesic flow of the right invariant inner metric on the $\operatorname{Diff}^{s}\left(S^{1}\right) \widehat{\ltimes C^{\infty}}\left(S^{1}\right)^{k+1}$, which at the identity is given by the $L^{2}$ inner product.

\section{THE RESULT OF THE PAPER}

THEOREM 1.1. Let $t \mapsto \hat{c}$ be a curve in the $\operatorname{Diff}^{s}\left(\widehat{\left.S^{1}\right) \ltimes C^{\infty}}\left(S^{1}\right)^{k}\right.$. Let $\hat{c}=(e, \mathbf{e}, 0)$ be the initial point, directing to the vector $\hat{c}(0)=\left(u(x)(d / d x), \vec{v}(x), \Gamma_{0}\right)$, where $\Gamma_{0}=\left(\gamma_{0}^{1}, \vec{\gamma}_{0}^{2}\right.$, $\left.\gamma_{0}^{3}\right) \in \mathbb{R}^{k+2}$ and $\vec{v}(x)=\left(v_{1}(x), \ldots, v_{k}(x)\right)^{t}$. Then, $\hat{c}(t)$ is a geodesic of the $L^{2}$ metric:

(A) to a hyperplane $\gamma_{0}^{1}=-1$, associated $\vec{\gamma}_{0}^{2}=\mathbf{0}, \gamma_{0}^{3}=0$ if and only if $(u(x, t)(d / d x)$, $\vec{v}(x, t), \Gamma)$ satisfies the multicomponent Ito-type system,

(B) associated to a hyperplane $\vec{\gamma}_{0}^{2}=\mathrm{Id}, \gamma_{0}^{1}=\gamma_{0}^{3}=0$ if and only if $(u(x, t)(d / d x)$, $\vec{v}(x, t), \Gamma)$ satisfies modified multicomponent dispersive water wave equation,

(C) associated to a hyperplane $\gamma_{0}^{1}=-1, \vec{\gamma}_{0}^{2}=\mathrm{Id}, \gamma_{0}^{3}=0$ if and only if $(u(x, t)(d / d x)$, $\vec{v}(x, t), \Gamma)$ satisfies some generalized multicomponent integrable system (stated in Section 4),

(D) associated to a hyperplane $\gamma_{0}^{1}=\gamma_{0}^{2}=\gamma_{0}^{3}=0$ if and only if $(u(x, t)(d / d x), \vec{v}(x, t)$, Г) satisfies modified multicomponent dispersionless long wave equations.

Next, we derive the Euler-Poincaré flows on the dual space of $\operatorname{Vect}\left(S^{1}\right) \ltimes C^{\infty+1}$. A typical element of this algebra is $\left(\begin{array}{c}f(x)(d / d x) \\ a(x) \\ \vec{p}\end{array}\right)$, where $f(x)(d / d x) \in \operatorname{Vect}\left(S^{1}\right), a(x) \in$ $C^{\infty}\left(S^{1}\right)$ and $\vec{p} \in C^{\infty}\left(S^{1}\right)^{k}$.

THEOREM 1.2. The geodesic flow of the right invariant inner product on the $\operatorname{Diff}\left(S^{1}\right) \ltimes$ $C^{\infty k+1}$, which at the identity is given by the $L^{2}$ metric, yields the multicomponent Ito-type systems.

2. Structure of $\operatorname{Vect}\left(S^{1}\right) \ltimes C^{\infty}\left(S^{1}\right)^{k}$. Let $\operatorname{Diff}^{s}\left(S^{1}\right)$ be the group of orientation preserving Sobolev $H^{s}$ diffeomorphisms of the circle. It is known that the group $\operatorname{Diff}^{S}\left(S^{1}\right)$ as well as its Lie algebra of vector fields on $S^{1}, T_{\mathrm{id}} \operatorname{Diff}^{\mathcal{S}}\left(S^{1}\right)=\operatorname{Vect}^{s}\left(S^{1}\right)$, have non-trivial one-dimensional central extensions, the Bott-Virasoro group $\widehat{\operatorname{Diff}}^{s}\left(S^{1}\right)$ and the Virasoro algebra Vir, respectively, [19, 20, 21, 27, 28].

The Lie algebra $\operatorname{Vect}^{s}\left(S^{1}\right)$ is the algebra of smooth vector fields on $S^{1}$. This satisfies the commutation relations

$$
\left[f \frac{d}{d x}, g \frac{d}{d x}\right]:=\left(f(x) g^{\prime}(x)-f^{\prime}(x) g(x)\right) \frac{d}{d x} .
$$


One parameter family of $\operatorname{Vect}^{s}\left(S^{1}\right)$ that acts on the space of all tensor densities on $S^{1}$ of degree $-\mu, a=a(x)(d x)^{-\mu}$, is given by

$$
L_{f(x)(d / d x)}^{(\mu)} \vec{a}(x)=f(x) \vec{a}^{\prime}(x)-\mu f^{\prime}(x) \vec{a}(x),
$$

where

$$
L_{f(x)(d / d x)}^{(\mu)}=f(x) \frac{d}{d x}-\mu f^{\prime}(x)
$$

is the Lie derivative with respect to the vector field $f(x)(d / d x)$ and

$$
\vec{a}=\left(a_{1}, \ldots, a_{k}\right)^{t} \in\left(C^{\infty}\left(S^{1}\right)\right)^{k} .
$$

The Lie algebra of Diff ${ }^{s}\left(S^{1}\right) \ltimes\left(C^{\infty}\left(S^{1}\right)\right)^{k}$ is the semidirect product Lie algebra

$$
\mathscr{G}=\operatorname{Vect}^{s}\left(S^{1}\right) \ltimes C^{\infty}\left(S^{1}\right)^{k} .
$$

An element of $\varphi$ is a pair $(f(x)(d / d x), \vec{a}(x))$, where $f(x)(d / d x) \in \operatorname{Vect}^{s}\left(S^{1}\right)$.

It is known that this algebra has a three-dimensional central extension given by the nontrivial cocycles

$$
\begin{aligned}
& \omega_{1}\left(\left(f \frac{d}{d x}, \vec{a}\right),\left(g \frac{d}{d x}, \vec{b}\right)\right)=\int_{S^{1}} f^{\prime}(x) g^{\prime \prime}(x) d x, \\
& \vec{\omega}_{2}\left(\left(f \frac{d}{d x}, \vec{a}\right),\left(g \frac{d}{d x}, \vec{b}\right)\right)=\int_{S^{1}} f^{\prime \prime}(x) \vec{b}(x)-g^{\prime \prime}(\vec{a}(x)) d x, \\
& \omega_{3}\left(\left(f \frac{d}{d x}, \vec{a}\right),\left(g \frac{d}{d x}, \vec{b}\right)\right)=2 \int_{S^{1}} \vec{a}^{t}(x) \vec{b}^{\prime}(x) d x .
\end{aligned}
$$

The first cocycle $\omega_{1}$ is the well-known Gelfand-Fuchs cocycle, and the second cocycle $\vec{\omega}_{2}$ takes its values in $\mathbb{R}^{k}$. The Virasoro algebra is the unique nontrivial central extension of $\operatorname{Vect}\left(S^{1}\right)$ via this $\omega_{1}$ cocycle. Hence, we define the Virasoro algebra

$$
\operatorname{Vir}=\operatorname{Vect}^{s}\left(S^{1}\right) \oplus \mathbb{R} .
$$

The space $C^{\infty}\left(S^{1}\right) \oplus \mathbb{R}$ is identified with a part of the dual space to the Virasoro algebra. It is called the regular part, and the pairing between this space and the Virasoro algebra is given by

$$
\left\langle(u(x), a),\left(f(x) \frac{d}{d x}, \alpha\right)\right\rangle=\int_{S^{1}} u(x) f(x) d x+a \alpha .
$$

Similarly, we consider an extension of $\mathscr{G}$. This extended algebra is given by

$$
\hat{\mathscr{G}}=\operatorname{Vect}^{s}\left(S^{1}\right) \ltimes\left(C^{\infty}\left(S^{1}\right)\right)^{k} \oplus \mathbb{R}^{k+2} .
$$

The Lie algebra $\hat{\mathscr{G}}$, for $k=1$, has been considered in various places [3, 15, 24]. It was shown in [24] that the cocycles

$$
H^{2}\left(\operatorname{Vect}\left(S^{1}\right) \ltimes\left(C^{\infty}\left(S^{1}\right)\right)\right)=\mathbb{R}^{3}
$$

define the universal central extension the Lie algebra $\operatorname{Vect}^{s}\left(S^{1}\right) \ltimes C^{\infty}\left(S^{1}\right)$. 
DEFINITION 2.1. The commutation relation in $\hat{\mathscr{G}}$ is given by

$$
\left[\left(\begin{array}{c}
f(x) \frac{d}{d x} \\
\vec{a} \\
\alpha
\end{array}\right),\left(\begin{array}{c}
g(x) \frac{d}{d x} \\
\vec{b} \\
\beta
\end{array}\right)\right]=\left(\begin{array}{c}
\left(f g^{\prime}-f^{\prime} g\right) \frac{d}{d x} \\
f \vec{b}^{\prime}-g \vec{a}^{\prime} \\
\omega
\end{array}\right),
$$

where $\boldsymbol{\alpha}=\left(\alpha_{1}, \vec{\alpha}_{2}, \alpha_{3}\right), \boldsymbol{\beta}=\left(\beta_{1}, \vec{\beta}_{2}, \beta_{3}\right) \in \mathbb{R}^{3}, \boldsymbol{\omega}=\left(\omega_{1}, \vec{\omega}_{2}, \omega_{3}\right)$ are the two cocycles.

The dual space of smooth functions

$$
C^{\infty}\left(S^{1}\right) \oplus \underbrace{C^{\infty}\left(S^{1}\right) \oplus \cdots C^{\infty}\left(S^{1}\right)}_{k}
$$

is the space of distributions (generalized functions) on $S^{1}$. Of particular interest are the orbits in the regular dual $\hat{G}_{\text {reg }}^{*}$. In the case of current group, Gelfand, Vershik, and Graev have constructed some of the corresponding representations.

DEFINITION 2.2. The regular part of the dual space $\hat{\mathscr{G}}^{*}$ to the Lie algebra $\hat{\mathscr{G}}$ is as follows. Consider

$$
\hat{\mathscr{G}}_{\mathrm{reg}}^{*}=C^{\infty}\left(S^{1}\right) \oplus \underbrace{C^{\infty}\left(S^{1}\right) \oplus \cdots \oplus C^{\infty}\left(S^{1}\right)}_{k} \oplus \mathbb{R}^{3}
$$

and fix the pairing between this space and $\hat{\mathscr{G}},\langle\cdot, \cdot\rangle: \hat{\mathscr{G}}_{\mathrm{reg}}^{*} \otimes \hat{\mathscr{G}} \rightarrow \mathbb{R}$ :

$$
\langle\hat{u}, \hat{f}\rangle=\int_{S^{1}} f(x) u(x) d x+\int_{S^{1}} \vec{v}^{t}(x) \vec{a}(x) d x+\boldsymbol{\alpha} \cdot \boldsymbol{\gamma},
$$

where $\hat{u}=\left(u(x), \vec{v}^{t}, \boldsymbol{\gamma}\right), \hat{f}=(f(d / d x), \vec{a}, \boldsymbol{\alpha})$.

Extend (2.14) to a right invariant metric on the semidirect product space $\operatorname{Diff}^{s}\left(\widehat{\left.S^{1}\right) \ltimes C^{\infty}}\left(S^{1}\right)^{k}\right.$ by setting

$$
\langle\hat{u}, \hat{f}\rangle_{\hat{\xi}}=\left\langle d_{\hat{\xi}} R_{\hat{\xi}^{-1}} \hat{u}, d_{\hat{\xi}} R_{\hat{\xi}^{-1}} \hat{f}\right\rangle_{L^{2}}
$$

for any $\hat{\xi} \in \hat{\mathscr{G}}$ and $\hat{u}, \hat{f} \in T_{\hat{\xi}} \hat{\mathscr{G}}$, where

$$
R_{\hat{\xi}}: \hat{G} \longrightarrow \hat{\mathscr{G}}
$$

is the right translation by $\hat{\xi}$.

3. Projective connections on the circle. In this section, we describe the matrix projective connection associated to $\operatorname{Vect}^{s}\left(S^{1}\right) \ltimes C^{\infty}\left(S^{1}\right)^{k}$ algebra. We start with some definitions of projective connections [12, 13, 14, 17].

We denote $\Omega^{ \pm 1 / 2}$ by the square root of the tangent and cotangent bundle of $S^{1}$, respectively.

Let $\Delta$ be a second-order differential operator. 
Definition 3.1. A projective connection on the circle is a linear second-order differential operator

$$
\Delta: \Gamma\left(\Omega^{-1 / 2}\right) \longrightarrow \Gamma\left(\Omega^{3 / 2}\right)
$$

such that

(1) the symbol of $\Delta$ is the identity,

(2)

$$
\int_{S^{1}}\left(\Delta s_{1}\right) s_{2}=\int_{S^{1}} s_{1}\left(\Delta s_{2}\right)
$$

for all $s_{i} \in \Gamma\left(\Omega^{-1 / 2}\right)$.

We take $s=\psi(x) d x^{-1 / 2} \in \Gamma\left(\Omega^{-1 / 2}\right)$, then $\Delta s \in \Gamma\left(\Omega^{3 / 2}\right)$ is locally described by

$$
\Delta s=\left(a \psi^{\prime \prime}+b \psi^{\prime}+c \psi\right) d x^{3 / 2}
$$

As discussed in [6], any differential equation of the form

$$
\frac{d^{2} y}{d x^{2}}=p_{3}\left(\frac{d y}{d x}\right)^{3}+p_{2}\left(\frac{d y}{d x}\right)^{2}+p_{1}\left(\frac{d y}{d x}\right)+p_{0}
$$

defines a projective structure.

From the definition of the projective connection condition (1) implies $a=1$ and condition (2) implies $b=0$, hence projective connection can be identified with the Hill operator

$$
\Delta^{(2)} \equiv \Delta=\frac{d^{2}}{d x^{2}}+u(x) .
$$

Let $L_{v}$ be the Lie derivative with respect to the vector field $v=f(x)(d / d x)$.

DEFINITION 3.2. A vector field is called projective vector field which keeps fixed a given projective connection $\Delta$

$$
\mathscr{L}_{v} \Delta s=\Delta\left(\mathscr{L}_{v} s\right)
$$

Using the equation $L_{v} \Delta^{(2)}=\Delta^{(2)} L_{v}$, we obtain the following proposition.

Proposition 3.3. A projective vector field $v=f(d / d x) \in \Gamma\left(\Omega^{-1}\right)$ satisfies

$$
f^{\prime \prime \prime}+4 f^{\prime} u+2 f u^{\prime}=0
$$

If $u_{i}$ are periodic functions on the line, the operator

$$
\frac{d^{n}}{d x^{n}}+u_{n-1} \frac{d^{n-1}}{d x^{n-1}}+u_{n-2} \frac{d^{n-2}}{d x^{n-2}}+u_{1} \frac{d}{d x}+u_{0}
$$


acting on periodic functions, is called an Adler-Gelfand-Dikii (or AGD) operator. We consider a projective connection as defined by this $n$ th-order operator on the circle $[12,13,14]$.

DEFINITION 3.4 (extended projective connection). An extended projective connection on the circle is a class of differential (conformal) operators

$$
\Delta^{(n)}: \Gamma\left(\Omega^{-(n-1) / 2}\right) \longrightarrow \Gamma\left(\Omega^{(n+1) / 2}\right)
$$

such that

(1) the symbol of $\Delta^{(n)}$ is the identity,

(2)

$$
\int_{S^{1}}\left(\Delta^{(n)} s_{1}\right) s_{2}=\int_{S^{1}} s_{1}\left(\Delta^{(n)} s_{2}\right)
$$

for all $s_{i} \in \Gamma\left(\Omega^{-(n-1) / 2}\right)$.

It is known that the symbol of an $n$ th-order operator from a vector bundle $U$ to $V$ is a section of $\operatorname{Hom}\left(U, V \otimes \operatorname{Sym}^{n} T\right)$, where

$$
U=\Omega^{-(n-1) / 2} V=\Omega^{(n+1) / 2} .
$$

Since $T=\Omega^{-1}$, we get

$$
V \otimes \operatorname{Sym}^{n} T \cong U,
$$

giving an invariant meaning to the first condition.

If $s_{2} \in \Gamma\left(\Omega^{-(n-1) / 2}\right)$, then $s_{1} \Delta^{(n)} s_{2} \in \Gamma(\Omega)$ is a one form to integrate.

The consequence of the first condition is that all the differential operators are monic, that is, the coefficient of the highest derivative is always one, and the second condition says that the term $u_{n-1}=0$.

3.1. Matrix projective connection. We first consider the projective connections yielded by the $\operatorname{Vect}\left(S^{1}\right) \ltimes C^{\infty}\left(S^{1}\right)$. Consider the following matrix linear differential operators on $C^{\infty}\left(S^{1}\right) \oplus C^{\infty}\left(S^{1}\right)$ :

$$
\Delta=\left(\begin{array}{cc}
\frac{d^{2}}{d x^{2}}+u(x) & \frac{d}{d x}+v(x) \\
\frac{d}{d x}+v(x) & c
\end{array}\right)
$$

where $c \in \mathbb{R}, u(x)=u(x+2 \pi)$ and $v(x)=v(x+2 \pi)$.

It defines a matrix projective connection. 
DEFINITION 3.5 (matrix projective connection). An extended projective connection on the circle is a class of differential (conformal) operators

$$
\Delta: \Gamma\left(\Omega^{-1 / 2}\right) \oplus \Gamma\left(\Omega^{1 / 2}\right) \longrightarrow \Gamma\left(\Omega^{3 / 2}\right) \oplus \Gamma\left(\Omega^{1 / 2}\right)
$$

such that

(1) the symbol of $\Delta$ is the identity,

(2)

$$
\int_{S^{1}} \vec{s}^{t} \Delta \vec{s}
$$

where $\vec{s}^{t}=\left(s_{1} s_{2}\right)$, for $s_{1} \in \Gamma\left(\Omega^{-1 / 2}\right)$ and $s_{2} \in \Gamma\left(\Omega^{1 / 2}\right)$, is a one form which can be integrated over the circle.

The second condition says that no choice of measure is necessary to make it invariant. Hence, the definition of a projective connection requires no structure on the circle other than a differentiable one. Thus, the space of matrix differential operator on a circle is considered as a module over the group of diffeomorphisms. In other words, the group of diffeomorphisms acts naturally on the projective structures.

The above construction can be easily generalized to the higher-dimensional matrix linear differential operators acting on

$$
\begin{gathered}
C^{\infty}\left(S^{1}\right) \oplus \underbrace{C^{\infty}\left(S^{1}\right) \oplus \cdots \oplus C^{\infty}\left(S^{1}\right)}_{k}, \\
\Delta_{\mathbf{k}+\mathbf{1}}=\left(\begin{array}{cc}
\frac{d^{2}}{d x^{2}}+u(x) & \vec{c}^{t} \frac{d}{d x}+\vec{v}^{t}(x) \\
\vec{c} \frac{d}{d x}+\vec{v}(x) & c_{1}
\end{array}\right),
\end{gathered}
$$

where $c_{1} \in \mathbb{R}, \vec{c} \in \mathbb{R}^{k}$, and $u(x)=u(x+2 \pi), \vec{v}(x)=\left(v_{1}, \ldots, v_{k}\right)^{t}=\vec{v}(x+2 \pi)$.

3.2. $\operatorname{Vect}\left(S^{1}\right) \ltimes C^{\infty}\left(S^{1}\right)^{k}$-module structure and projective vector field. A one-parameter family of $\operatorname{Vect}\left(S^{1}\right) \ltimes C^{\infty}\left(S^{1}\right)^{k}$ modules on the space $C^{\infty}\left(S^{1}\right) \oplus \underbrace{C^{\infty}\left(S^{1}\right) \oplus \cdots \oplus C^{\infty}\left(S^{1}\right)}_{k}$
is defined as follows:

$$
T_{(f(x)(d / d x), \vec{a}(x))}^{\lambda}\left(\begin{array}{c}
m \\
\vec{n}
\end{array}\right)=\left(\begin{array}{c}
L_{f(x)(d / d x)}^{\lambda} m \\
L_{f(x)(d / d x)}^{\lambda-1} \vec{n}-\lambda \vec{a}^{\prime}(x) m(x)
\end{array}\right),
$$

where $m(x) \in C^{\infty}\left(S^{1}\right)$ and $\vec{n}(x) \in C^{\infty}\left(S^{1}\right)^{k}$.

DEFINITION 3.6. The $\operatorname{Vect}\left(S^{1}\right) \ltimes C^{\infty}\left(S^{1}\right)^{k}$ action on the space of operators $\Delta_{\mathbf{k}+\mathbf{1}}$ is given by

$$
\left[T_{(f(x)(d / d x), \vec{a}(x))}, \Delta_{\mathbf{k}+\mathbf{1}}\right]=T_{(f(x)(d / d x), \vec{a}(x))}^{-1 / 2} \circ \Delta_{\mathbf{k}+\mathbf{1}}-\Delta_{\mathbf{k}+\mathbf{1}} \circ T_{(f(x)(d / d x), \vec{a}(x))}^{1 / 2} .
$$




\section{Claim.}

$$
\left[T_{f(x)(d / d x), \vec{a}(x)}^{\lambda}, T_{f(x)(d / d x), \vec{b}(x)}^{\lambda}\right]=T_{\left(\left(f g^{\prime}-f^{\prime} g\right)(d / d x), f \vec{b}^{\prime}-g \vec{a}^{\prime}\right)}^{\lambda}
$$

SKETCH OF THE PROOF. It is easy to compute from (3.17) that

$$
\begin{aligned}
& T_{g(x)(d / d x), \vec{b}(x)}^{\lambda} T_{f(x)(d / d x), \vec{a}(x)}^{\lambda}\left(\begin{array}{c}
m \\
\vec{n}
\end{array}\right) \\
& \quad=\left(\begin{array}{c}
L_{g(x)(d / d x)}^{\lambda} L_{f(x)(d / d x)}^{\lambda} m \\
L_{g(x)(d / d x)}^{\lambda-1} L_{f(x)(d / d x)}^{\lambda-1} \vec{n}-L_{g(x)(d / d x)}^{\lambda-1}\left(\lambda \vec{a}^{\prime}(x) m(x)\right)-\lambda \vec{b}^{\prime}(x) L_{f(x)(d / d x)}^{\lambda} m(x)
\end{array}\right), \\
& T_{f(x)(d / d x), \vec{a}(x)}^{\lambda} T_{g(x)(d / d x), \vec{b}(x)}^{\lambda}\left(\begin{array}{c}
m \\
\vec{n}
\end{array}\right) \\
& \quad=\left(\begin{array}{c}
L_{f(x)(d / d x)}^{\lambda} L_{g(x)(d / d x)}^{\lambda} m \\
L_{f(x)(d / d x)}^{\lambda-1} L_{g(x)(d / d x)}^{\lambda-1} \vec{n}-L_{f(x)(d / d x)}^{\lambda}\left(\lambda \vec{a}^{\prime}(x) m(x)\right)-(\lambda-1) \vec{b}^{\prime}(x) L_{g(x)(d / d x)}^{\lambda} m(x)
\end{array}\right) .
\end{aligned}
$$

Our result follows from (3.20).

A projective vector field in this case is a vector field $v=f(x)(d / d x)$ which leaves the projective connection invariant, that is,

$$
T_{(f(x)(d / d x), \vec{a}(x))}^{\lambda} \Delta_{\mathbf{k}+\mathbf{1}} \vec{s}=\Delta_{\mathbf{k}+\mathbf{1}} T_{(f(x)(d / d x), \vec{a}(x))}^{\lambda} \vec{s}
$$

for all $\vec{s}=\left(s_{1}, s_{2}\right)^{t} \in \Gamma\left(\Omega^{-1 / 2}\right) \oplus \Gamma\left(\Omega^{1 / 2}\right)$.

\section{Proposition 3.7.}

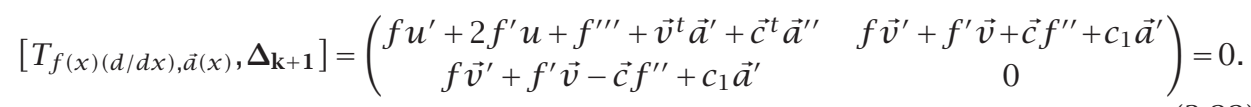

Proof. By direct calculation.

4. Integrable geodesic flows on $\operatorname{Diff}\left(S^{1}\right) \ltimes C^{\infty}\left(S^{1}\right)^{k}$. Let $G$ be a Lie group and $\mathfrak{g}$ its corresponding Lie algebra and its dual is denoted by $\mathfrak{g}^{*}$.

The dual space $\mathfrak{g}^{*}$ to any Lie algebra $\mathfrak{g}$ carries a natural Lie-Poisson structure [4, 7]:

$$
\{f, g\}_{\mathrm{LP}}(\mu):=\langle[d f, d g], \mu\rangle
$$

for any $\mu \in \mathfrak{g}^{*}$ and $f, g \in C^{\infty}\left(S^{1}\right)$.

LEMMA 4.1. The Hamiltonian vector field on $\mathfrak{g}^{*}$ corresponding to a Hamiltonian function $f$, computed with respect to the Lie-Poisson structure, is given by

$$
\frac{d \mu}{d t}=a d_{d f}^{*} \mu .
$$


Proof. The proof follows from the following identities:

$$
\left.i_{X_{f}} d g\right|_{\mu}=\left.L_{X_{f}} g\right|_{\mu}=\{f, g\}_{\mathrm{LP}}(\mu)=\langle[d g, d f], \mu\rangle=\left\langle d g, a d_{d f}^{*} \mu\right\rangle .
$$

This implies that $X_{f}=a d_{d f}^{*} \mu$. Thus, the Hamiltonian equation $d \mu / d t=X_{f}$ yields our result.

Let $I$ be an inertia operator

$$
I: \mathfrak{g} \longrightarrow \mathfrak{g}^{*}
$$

and then $\mu \in \mathfrak{g}^{*}$ evolve by

$$
\frac{d \mu}{d t}=\left(I^{-1} \mu\right) \cdot \mu
$$

where right-hand side denotes the coadjoint action of $\mathfrak{g}$ on $\mathfrak{g}^{*}$. This equation is called the Euler-Poincaré equation corresponding to the Hamiltonian $H(\mu)=(1 / 2)\left\langle I^{-1} \mu, \mu\right\rangle$.

The Euler-Poincaré equation is the Hamiltonian flow on the coadjoint orbits on the dual of Bott-Virasoro algebra generated by the Hamiltonian

$$
H\left(q \partial_{x}, a\right)=\frac{1}{2} \int_{S^{1}} q^{2} d x+a^{2}
$$

where $a$ is just a constant.

Proposition 4.2. Let $\Omega G$ be an infinite-dimensional Lie group equipped with a right invariant metric. A curve $t \rightarrow c(t)$ in $\Omega G$ is a geodesic of this metric if and only if $q(t)=$ $d_{c_{t}} R_{c_{t}^{-1}} \dot{c}(t)$ satisfies

$$
\frac{d}{d t} q(t)=-a d_{q(t)}^{*} q(t)
$$

Given any three elements $\hat{f}, \hat{g}$, and $\hat{u}$,

$$
\hat{f}=\left(f \frac{d}{d x}, \vec{a}, \boldsymbol{\alpha}\right), \quad \hat{g}=\left(g \frac{d}{d x}, \vec{b}, \boldsymbol{\beta}\right), \quad \hat{u}=\left(u \frac{d}{d x}, \vec{v}, \mathbf{c}\right)
$$

in $\hat{\mathscr{G}}$, where $\boldsymbol{\alpha}=\left(\alpha_{1}, \vec{\alpha}_{2}, \alpha_{3}\right), \boldsymbol{\beta}=\left(\beta_{1}, \vec{\beta}_{2}, \beta_{3}\right), \mathbf{c}=\left(c_{1}, \vec{c}_{2}, c_{3}\right)$.

LEMMA 4.3.

$$
\operatorname{ad}_{\hat{f}}^{*} \hat{u}=\left(\begin{array}{c}
2 f^{\prime}(x) u(x)+f(x) u^{\prime}(x)+\vec{a}^{\prime} v(x)-c_{1} f^{\prime \prime \prime}+\vec{c}_{2}^{t} \vec{a}^{\prime \prime} \\
f^{\prime} \vec{v}(x)+f(x) \vec{v}^{\prime}(x)-\vec{c}_{2} f^{\prime \prime}(x)+2 c_{3} \vec{a}^{\prime}(x) \\
0
\end{array}\right)
$$


Proof. This proof follows from

$$
\begin{aligned}
\left\langle a d_{\hat{f}}^{*} \hat{u}, \hat{g}\right\rangle_{L^{2}}= & \langle\hat{u},[\hat{f}, \hat{g}]\rangle_{L^{2}} \\
= & \left\langle\left(u(x) \frac{d}{d x}, \vec{v}(x), c\right),\left[\left(f g^{\prime}-f^{\prime} g\right) \frac{d}{d x}, f \vec{b}^{\prime}-g \vec{a}^{\prime}, \omega\right]\right\rangle_{L^{2}} \\
= & -\int_{S^{1}}\left(f g^{\prime}-f^{\prime} g\right) u(x) d x-\int_{S^{1}}\left(f \vec{b}^{\prime}-g \vec{a}^{\prime}\right) v d x-c_{1} \int_{S^{1}} f^{\prime}(x) g^{\prime \prime}(x) d x \\
& -c_{2} \int_{S^{1}}\left(f^{\prime \prime}(x) \vec{b}(x)-g^{\prime \prime}(x) \vec{a}(x)\right) d x-2 c_{3} \int_{S^{1}} \vec{a}^{t}(x) \vec{b}^{\prime}(x) d x .
\end{aligned}
$$

Since $f, g, u$ are periodic functions, integrating by parts we obtain our result.

CASE 1 (the multicomponent Ito equation). The coadjoint action leaves the parameter space invariant. We consider a hyperplane $c_{1}=-1, \vec{c}_{2}=c_{3}=0$.

LEMMA 4.4.

$$
a d_{\hat{f}}^{*} \hat{u}=\left(\begin{array}{c}
2 f^{\prime}(x) u(x)+f(x) u^{\prime}(x)+\vec{a}^{t^{\prime}}(x) \vec{v}(x)+f^{\prime \prime \prime} \\
f^{\prime} \vec{v}(x)+f(x) \vec{v}^{\prime}(x) \\
0
\end{array}\right) .
$$

The Euler-Poincaré equation is the Hamiltonian flow on the coadjoint orbit in $\hat{\varphi}^{*}$, generated by the Hamiltonian

$$
H(\hat{u}) \equiv H(u, v)=\langle(u(x), \vec{v}(x)),(u(x), \vec{v}(x))\rangle,
$$

given by

$$
\frac{d \hat{u}}{d t}=-a d_{\hat{u}(t)}^{*} u(t)
$$

Let $V$ be a vector space and assume that the Lie group $G$ acts on the left by linear maps on $V$, thus $G$ acts on the left on its dual space $V^{*}$ [for details, see, e.g., [7]].

Proposition 4.5. Let $G \ltimes V$ be a semidirect product space (possibly infinite dimensional), equipped with a metric $\langle\cdot, \cdot\rangle$ which is right translation. A curve $t \rightarrow c(t)$ in $G \ltimes V$ is a geodesic of this metric if and only if $u(t)=d_{c(t)} R_{c(t)^{-1}} \dot{c}(t)$ satisfies the Euler-Poincaré equation.

Thus, we obtain the multicomponent Ito equation

$$
\begin{gathered}
u_{t}+u_{x x x}+6 u u_{x}+2 \vec{v}^{t} \vec{v}_{x}=0, \\
\vec{v}_{t}+2(u \vec{v})_{x}=0 .
\end{gathered}
$$

The original Ito system admits a tri-Hamiltonian structure, hence, this multicomponent Ito would exhibit the same property.

CASE 2 (modified multicomponent dispersive water wave equation). When we restrict to a hyperplane $c_{1}=0, c_{3}=0$, we obtain the following lemma. 
LEMMA 4.6.

$$
\operatorname{ad}_{\hat{f}}^{*} \hat{u}=\left(\begin{array}{c}
2 f^{\prime}(x) u(x)+f(x) u^{\prime}(x)+\vec{a}^{\prime}(x) \vec{v}(x)+\vec{c}_{2}^{t} \vec{a}^{\prime \prime} \\
f^{\prime} \vec{v}(x)+f(x) \vec{v}^{\prime}(x)-\vec{c}_{2} f^{\prime \prime} \\
0
\end{array}\right) .
$$

Thus, by applying the Euler-Poincaré equation we obtain

$$
\begin{gathered}
u_{t}+6 u u_{x}+2 \vec{v}^{t} \vec{v}_{x}+\vec{c}_{2}^{t} \vec{v}_{x x}=0, \\
\vec{v}_{t}+2(\vec{v} u)_{x}-\vec{c}_{2} u_{x x}=0 .
\end{gathered}
$$

CASE 3 (the multicomponent new integrable system). When we restrict to a hyperplane $c_{1}=1, c_{3}=0$, we obtain the following lemma.

\section{LEMMA 4.7 .}

$$
a d_{\hat{f}}^{*} \hat{u}=\left(\begin{array}{c}
2 f^{\prime}(x) u(x)+f(x) u^{\prime}(x)+a^{\prime} v(x)+a^{\prime \prime}+f^{\prime \prime \prime} \\
f^{\prime} v(x)+f(x) v^{\prime}(x)-f^{\prime \prime} \\
0
\end{array}\right)
$$

Thus, by applying the Euler-Poincaré equation, we obtain another pair of integrable Hamiltonian system

$$
\begin{gathered}
u_{t}+6 u u_{x}+2 \vec{v}^{t} \vec{v}_{x}+\vec{c}_{2}^{t} \vec{v}_{x x}+u_{x x x}=0, \\
\vec{v}_{t}+2(\vec{v} u)_{x}-\vec{c}_{2} u_{x x}=0 .
\end{gathered}
$$

CASE 4 (modified multicomponent dispersionless long wave equation). In this case, we just set $c_{1}=c_{2}=c_{3}=0$, that is, non-centrally extended part.

LEMMA 4.8.

$$
a d_{\hat{f}}^{*} \hat{u}=\left(\begin{array}{c}
2 f^{\prime}(x) u(x)+f(x) u^{\prime}(x)+\vec{a}^{\prime}(x) \vec{v}(x) \\
f^{\prime} \vec{v}(x)+f(x) \vec{v}^{\prime}(x) \\
0
\end{array}\right)
$$

Thus, we obtain

$$
\begin{gathered}
u_{t}+6 u u_{x}+2 \vec{v}^{t} \vec{v}_{x}=0, \\
\vec{v}_{t}+2(\vec{v} u)_{x}=0 .
\end{gathered}
$$

5. Second category of multiple-component integrable systems. In this section, we consider the Euler-Arnold equation on the dual space of $\operatorname{Vect}\left(S^{1}\right) \ltimes C^{\infty}\left(S^{1}\right)^{k+1}$. We split the space $C^{\infty}\left(S^{1}\right)^{k+1}$ into $C^{\infty}\left(S^{1}\right) \times C^{\infty}\left(S^{1}\right)^{k}$. A typical element of $\operatorname{Vect}\left(S^{1}\right) \ltimes C^{\infty}\left(S^{1}\right)^{k+1}$ is $\left(\begin{array}{c}f(x)(d / d x) \\ a(x) \\ \vec{p}\end{array}\right)$. This type of construction has been discussed by Kupershmidt [23]. 
We redefine the cocycles in the following form:

$$
\begin{aligned}
& \omega_{1}\left(\left(f \frac{d}{d x}, a, \vec{p}\right),\left(g \frac{d}{d x}, b, \vec{q}\right)\right)=\int_{S^{1}} f^{\prime}(x) g^{\prime \prime}(x) d x, \\
& \omega_{2}\left(\left(f \frac{d}{d x}, a, \vec{p}\right),\left(g \frac{d}{d x}, b, \vec{q}\right)\right)=\int_{S^{1}}\left(f^{\prime \prime}(x) b(x)-g^{\prime \prime} a(x)\right) d x, \\
& \omega_{3}\left(\left(f \frac{d}{d x}, a, \vec{p}\right),\left(g \frac{d}{d x}, b, \vec{q}\right)\right)=2 \int_{S^{1}} a(x) b^{\prime}(x) d x .
\end{aligned}
$$

This means

$$
H^{2}\left(\operatorname{Vect}\left(S^{1}\right) \ltimes C^{\infty}\left(S^{1}\right)^{k+1}\right)=\mathbb{R}^{3} .
$$

DEFINITION 5.1. The commutation relation in $\hat{\varphi}$ is given by

$$
\left[\left(\begin{array}{c}
f(x) \frac{d}{d x} \\
a(x) \\
\vec{p} \\
\boldsymbol{\alpha}
\end{array}\right),\left(\begin{array}{c}
\left.g(x) \frac{d}{d x}\right) \\
b(x) \\
\vec{q} \\
\boldsymbol{\beta}
\end{array}\right)\right]:=\left(\begin{array}{c}
\left(f g^{\prime}-f^{\prime} g\right) \frac{d}{d x} \\
f b^{\prime}-g a^{\prime} \\
f \vec{q}^{\prime}-g \vec{p}^{\prime} \\
\omega
\end{array}\right),
$$

where $\boldsymbol{\alpha}=\left(\alpha_{1}, \vec{\alpha}_{2}, \alpha_{3}\right), \boldsymbol{\beta}=\left(\beta_{1}, \overrightarrow{\beta_{2}}, \beta_{3}\right) \in \mathbb{R}^{3}, \boldsymbol{\omega}=\left(\omega_{1}, \vec{\omega}_{2}, \omega_{3}\right)$ are the two cocycles.

DEFINITION 5.2. The regular part of the dual space $\hat{\mathscr{G}}^{*}$ to the Lie algebra $\hat{\mathscr{G}}$ is as follows. Consider (2.13) and fix the pairing between this space and $\hat{\mathscr{G}},\langle\cdot, \cdot\rangle: \hat{\mathscr{G}}_{\mathrm{reg}}^{*} \otimes \hat{\mathscr{G}} \rightarrow \mathbb{R}$ :

$$
\langle\hat{u}, \hat{f}\rangle=\int_{S^{1}} f(x) u(x) d x+\int_{S^{1}} \vec{a}(x) \vec{v}(x) d x+\boldsymbol{\alpha} \cdot \boldsymbol{\gamma},
$$

where $\hat{u}=(u(x), v, \vec{w}, \boldsymbol{\gamma}), \hat{f}=(f(d / d x), a, \vec{p}, \boldsymbol{\alpha})$.

Again, from the coadjoint action, we obtain the following set of integrable Hamiltonian system:

$$
\begin{gathered}
u_{t}+6 u u_{x}+2 v v_{x}+v_{x x}+u_{x x x}=0, \\
v_{t}+2(v u)_{x}-u_{x x}=0, \\
\vec{w}_{t}+2(\vec{w} u)_{x}=0 .
\end{gathered}
$$

This is an avatar of the Ito equation. Similarly, we can derive the other sets of equations.

This equation admits a bi-Hamiltonian structure

$$
\mathbf{D}_{2} \delta H_{n}=\mathbf{D}_{1} \delta H_{n+1},
$$


where

$$
\mathbf{D}_{2}=\left(\begin{array}{ccc}
D^{3}+4 u D+2 u_{x} & 2 v D & 0 \\
2 v_{x}+2 v D & D^{2} & 0 \\
2 \vec{w}_{x}+2 \vec{w} D & 0 & 0
\end{array}\right), \quad \mathbf{D}_{1}=\left(\begin{array}{ccc}
D & 0 & 0 \\
0 & D & 0 \\
0 & 0 & D
\end{array}\right)
$$

with the Hamiltonian functionals

$$
\begin{aligned}
& H_{1}[u, v, \vec{w}]=\frac{1}{2} \int\left(u^{2}+v^{2}+\mathbf{w}^{2}\right) d x \\
& H_{2}[u, v, \vec{w}]=\frac{1}{2} \int\left(u^{3}+u v_{x}-\frac{1}{2} u_{x}^{2} \vec{w}+u v^{2}+u \mathbf{w}^{2}\right) d x
\end{aligned}
$$

The recursion operator arising from a Hamiltonian pair

$$
\mathbb{R}=\mathbf{D}_{2} \mathbf{D}_{1}^{-1}=\left(\begin{array}{ccc}
D^{2}+4 u+2 u_{x} D^{-1} & 2 v & 0 \\
2 v_{x} D^{-1}+2 v & D & 0 \\
2 \vec{w}_{x} D^{-1}+2 \vec{w} & 0 & 0
\end{array}\right)
$$

is a hereditary operator which yields infinitely many conserved quantities.

6. Conclusion and outlook. In this paper, we have continued to study a question posed by Marcel, Ovsienko, and Roger "what are the integrable systems associated to the coadjoint orbit of the extended Bott-Virasoro group?" In particular, in our earlier paper, we have identified a large class of two-component integrable systems associated to $\operatorname{Diff}\left(S^{1) \ltimes C}{ }^{\infty}\left(S^{1}\right)\right.$. These are mostly tri-Hamiltonian systems.

In this paper, we further extend the earlier group to $\operatorname{Diff}\left(\widehat{\left.S^{1}\right) \ltimes C}{ }^{\infty}\left(S^{1}\right)^{k}\right.$. We have shown that a large class of multicomponent integrable systems can be derived as a geodesic flow on this space. Some of the integrable systems have been already discussed by Marek Antonowicz and Allan Fordy and Kupershmidt. Thus, we study the multicomponent integrable systems from this geometric point of view.

In this paper, we have left out two more generalizations of this construction. The first one is the supersymmetric generalization to obtain the super multicomponent integrable systems, and the second one is the $H^{1}$ counterpart of the multicomponent integrable systems considered here.

Acknowledgments. The author is grateful to Professors Claude Roger and Valentin Ovsienko for their interest, encouragement, and many valuable remarks. He also thanks Professors Jerry Marsden, Boris Kupershmidt, and Richard Montgomery for their helpful discussions. He would like to thank Professor Maxim Kontsevich and the staffs of IHES, and Professor Dieter Mayer and Dr. Cheng-Hung Chang at Mathematische Physik, Technische Universität Clausthal, for their hospitality when the core part of the work was done. 


\section{REFERENCES}

[1] M. Antonowicz and A. P. Fordy, Coupled KdV equations with multi-Hamiltonian structures, Phys. D 28 (1987), no. 3, 345-357.

[2] _ A family of completely integrable multi-Hamiltonian systems, Phys. Lett. A 122 (1987), no. 2, 95-99.

[3] E. Arbarello, C. De Concini, V. G. Kac, and C. Procesi, Moduli spaces of curves and representation theory, Comm. Math. Phys. 117 (1988), no. 1, 1-36.

[4] V. I. Arnold, Mathematical Methods of Classical Mechanics, 2nd ed., Graduate Texts in Mathematics, vol. 60, Springer, New York, 1989.

[5] R. Camassa and D. D. Holm, An integrable shallow water equation with peaked solitons, Phys. Rev. Lett. 71 (1993), no. 11, 1661-1664.

[6] E. Cartan, Lecons sur la Theorie des Espaces a Connection Projective, Gauthier-Villars, Paris, 1937.

[7] H. Cendra, D. D. Holm, J. E. Marsden, and T. S. Ratiu, Lagrangian reduction, the EulerPoincaré equations, and semidirect products, Geometry of Differential Equations, Amer. Math. Soc. Transl. Ser. 2, vol. 186, American Mathematical Society, Rhode Island, 1998, pp. 1-25.

[8] D. G. Ebin and J. Marsden, Groups of diffeomorphisms and the notion of an incompressible fluid, Ann. of Math. (2) 92 (1970), 102-163.

[9] P. Guha, Euler-Poincaré Formalism of Coupled KdV type systems and Diffeomorphism group on $S^{1}$, submitted.

[10] - Integrable geodesic flows on the (super)extension of the Bott-Virasoro group, Lett. Math. Phys. 52 (2000), no. 4, 311-328.

[11] Ito equation as a geodesic flow on $\left(\operatorname{Diff}^{\mathcal{S}}\left(\widehat{S}^{1) \odot C} C^{\infty}\left(S^{1}\right)\right)\right.$, Arch. Math. (Brno) 36 (2000), no. 4, 305-312.

[12] _ Projective connections, AGD manifold and integrable systems, Rev. Math. Phys. 12 (2000), no. 10, 1391-1406.

[13] _ Diff $\left(S^{1}\right)$ and Adler-Gelfand-Dikii spaces and integrable systems, Lett. Math. Phys. 55 (2001), no. 1, 17-31.

[14] _ Diffeomorphisms on $S^{1}$, projective structures and integrable systems, ANZIAM J. 44 (2002), no. 1, 169-180.

[15] J. Harnad and B. A. Kupershmidt, Symplectic geometries on $T^{*} \tilde{G}$, Hamiltonian group actions and integrable systems, J. Geom. Phys. 16 (1995), no. 2, 168-206.

[16] R. Hirota and J. Satsuma, Soliton solutions of a coupled Korteweg-de Vries equation, Phys. Lett. A 85 (1981), no. 8-9, 407-408.

[17] N. Hitchin, Vector fields on the circle, Mechanics, Analysis and Geometry: 200 Years After Lagrange (M. Francaviglia, ed.), North-Holland Delta Ser., North-Holland Publishing, Amsterdam, 1991, pp. 359-378.

[18] M. Ito, Symmetries and conservation laws of a coupled nonlinear wave equation, Phys. Lett. A 91 (1982), no. 7, 335-338.

[19] C. Kapoudjian, Coadjoint orbits of extensions of $\operatorname{Diff}^{+}\left(S^{1}\right)$ by modules of tensor densities, J. Geom. Phys. 33 (2000), no. 1-2, 1-22.

[20] A. A. Kirillov, The orbits of the group of diffeomorphisms of the circle, and local Lie superalgebras, Funktsional. Anal. i Prilozhen. 15 (1981), no. 2, 75-76.

[21] _ Infinite-dimensional Lie groups: their orbits, invariants and representations. The geometry of moments, Twistor Geometry and Nonlinear Systems (Primorsko, 1980), Lecture Notes in Math., vol. 970, Springer, Berlin, 1982, pp. 101-123.

[22] B. A. Kupershmidt, Mathematics of dispersive water waves, Comm. Math. Phys. 99 (1985), no. $1,51-73$.

[23] - A multicomponent water wave equation, J. Phys. A 18 (1985), no. 18, L1119-L1122.

[24] P. Marcel, V. Ovsienko, and C. Roger, Extension of the Virasoro and Neveu-Schwarz algebras and generalized Sturm-Liouville operators, Lett. Math. Phys. 40 (1997), no. 1, 31-39. 
[25] G. Misiołek, A shallow water equation as a geodesic flow on the Bott-Virasoro group, J. Geom. Phys. 24 (1998), no. 3, 203-208.

[26] V. Yu. Ovsienko and B. A. Khesin, The super Korteweg-de Vries equation as an Euler equation, Funktsional. Anal. i Prilozhen. 21 (1987), no. 4, 81-82.

[27] V. Yu. Ovsienko and C. Roger, Extension of Virasoro group and Virasoro algebra by modules of tensor densities on $S^{1}, 30$ (1996), 290-291, Funct. Anal. Appl.

[28] G. Segal, Unitary representations of some infinite-dimensional groups, Comm. Math. Phys. 80 (1981), no. 3, 301-342.

[29] _ The geometry of the KdV equation. Topological methods in quantum field theory (Trieste, 1990), Internat. J. Modern Phys. A 6 (1991), no. 16, 2859-2869.

[30] G. Wilson, The affine Lie algebra $C_{2}^{(1)}$ and an equation of Hirota and Satsuma, Phys. Lett. A 89 (1982), no. 7, 332-334.

Partha Guha: Mathematische Physik, Institut für Theoretische Physik, Technische Universität Clausthal, Arnold Sommerfeld Straße 6, 38678 Clausthal-Zellerfeld, Germany

Current address: S.N. Bose National Centre for Basic Sciences, JD Block, Sector III, Calcutta 700098, India

E-mail address: partha@bose. res. in 


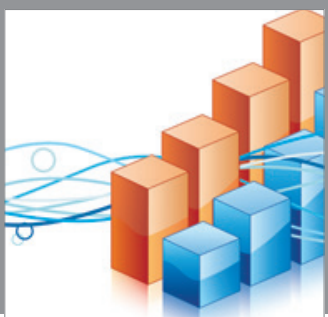

Advances in

Operations Research

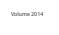

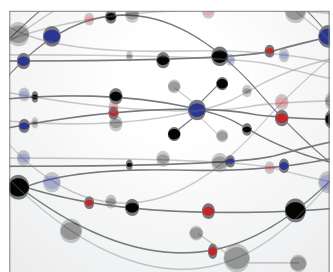

\section{The Scientific} World Journal
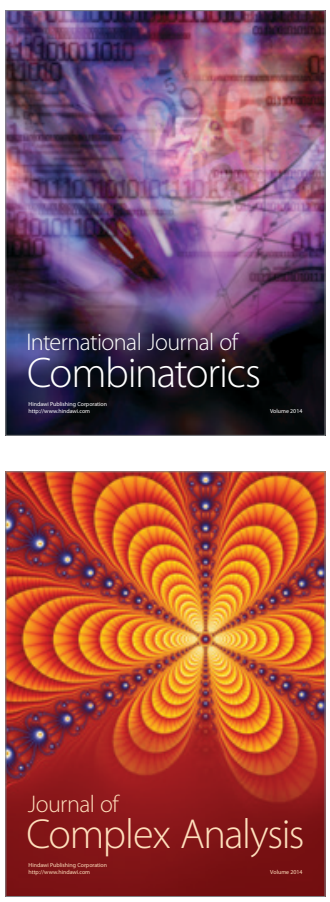

International Journal of

Mathematics and

Mathematical

Sciences
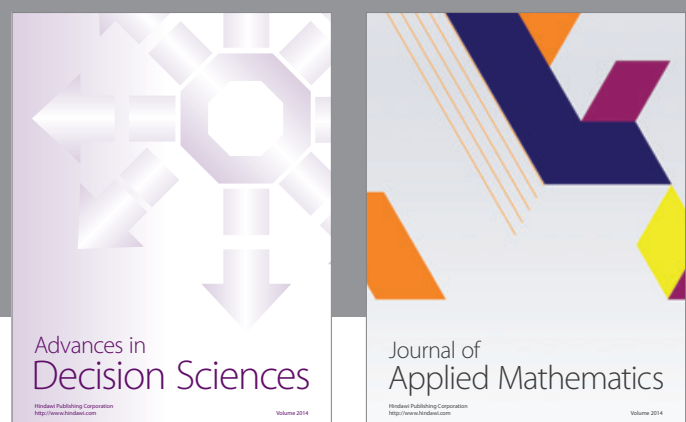

Journal of

Applied Mathematics
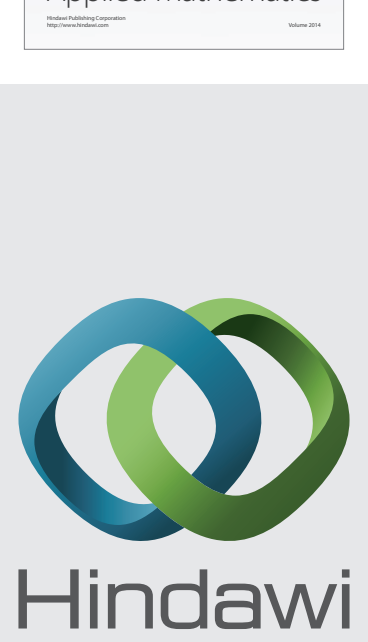

Submit your manuscripts at http://www.hindawi.com
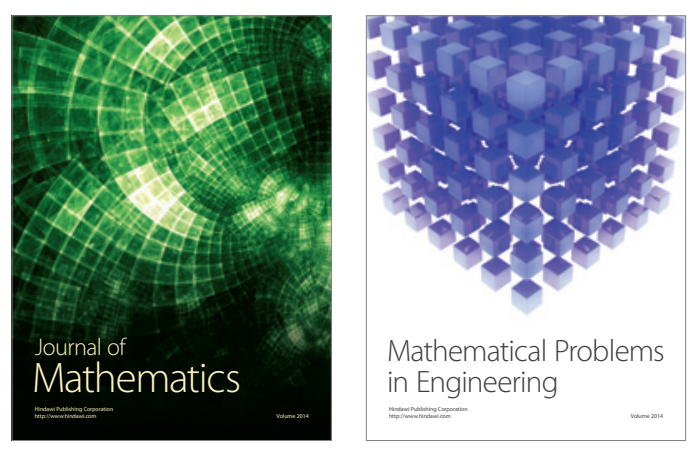

Mathematical Problems in Engineering
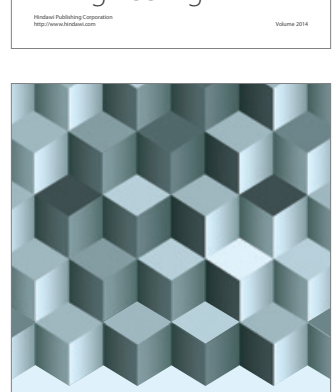

Journal of

Function Spaces
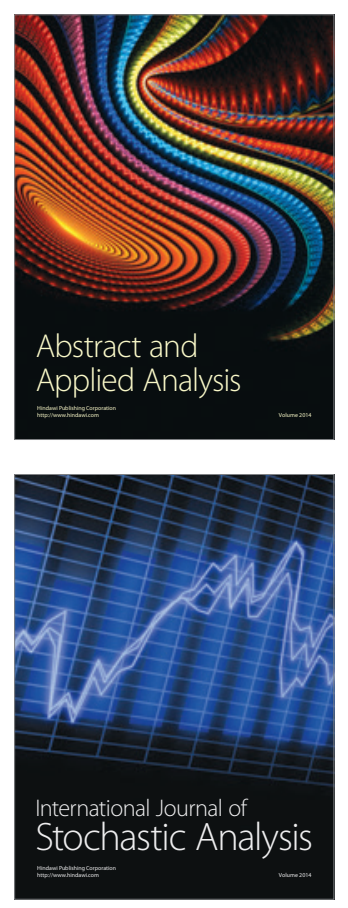

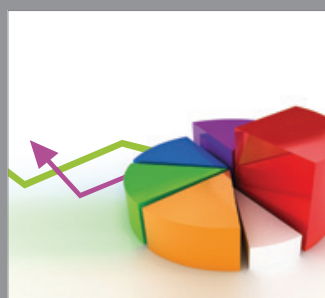

ournal of

Probability and Statistics

Promensencen
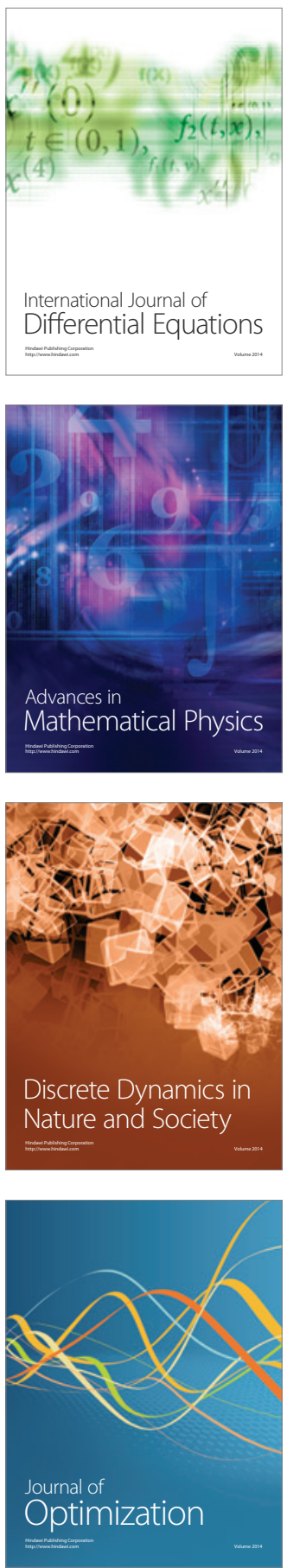